\title{
Investigating the FAIR Equivalency in National Guidance in Health in Kenya
}

\author{
Esther Thea INAU, ${ }^{\mathrm{a}, 1}$, Reginald NALUGALA ${ }^{\mathrm{b}, \mathrm{c}}$, William Muhadi NANDWA ${ }^{\mathrm{d}}$, \\ Fredrick OBWANDA ${ }^{\mathrm{c}}$, Antony WACHIRA ${ }^{\mathrm{e}}$, \\ Dagmar WALTEMATH ${ }^{\mathrm{a}}$, Antonio CARTAXO ${ }^{\mathrm{f}}$ and Atinkut ZELEKE ${ }^{\mathrm{a}}$ \\ a Department of Medical Informatics, University Medicine Greifswald, Germany \\ ${ }^{b}$ Institute of Social Transformation, Tangaza University, Kenya \\ ${ }^{c}$ VODAN-Africa, Kenya \\ ${ }^{d}$ Pumwani Hospital, Kenya \\ e Strathmore University, Kenya \\ ${ }^{f}$ The Globalisation, Accessibility, Innovation \& Care Network, \\ Leiden University, The Netherlands
}

Keywords. Data stewardship, FAIR, COVID-19, Kenya

\section{Introduction}

The growth in the public health sector in Sub-Saharan Africa has been supported by the implementation of various Health Information Systems [1]. The implementation of the FAIR data principles is advocated as an important cornerstone in research data management [2]. However, the implementation requires a comprehensive understanding of the already existing infrastructure and the demands of the implementing communities [3]. The Virus Outbreak Data Network (VODAN) project aims to integrate FAIRified data on the SARS CoV-2 virus in Sub-Saharan Africa (SSA) countries [4,5]. FAIR data principles are better implemented in Europe than in Africa, where there is hardly implementation at all [4]. Undefined data ownership in Africa is among the obstacles to comprehensive data stewardship during the COVID-19 pandemic [6]. This work explores the documented guidance authored by the governmental authorities in Kenya from 2006 to 2019 to direct the health and ICT sectors. We review the existent background regarding the policies, Acts, national strategies and national guidelines that may influence the uptake of the FAIR data principles in Kenya and further enable a FAIR digital data health infrastructure in Africa for reporting and research. The results serve to inform on the feasibility of FAIR implementaion within a framework of national relevance.

\section{Methods}

We conducted a qualitative cross-sectional study on 14 documents authored by the national authorities in Kenya from 2006 to 2019 to direct the health and ICT sectors.

\footnotetext{
${ }^{1}$ Corresponding Author, Esther Inau; E-mail: inaue@uni-greifswald.de.
} 
Here we measure the convergence between the FAIR data principles and the existing regulatory frameworks in Kenya's health data stewardship sector. We examined the document collection with respect to explicit mentions of the FAIR data principles. If no mention of FAIR had been found, the documents were further examined to determine direct mentions of the 15 FAIR data facets or of concepts representing them [4]. Our investigation is based on the "FAIR Equivalency" index, which indicates the degree of agreement between Kenya's national regulatory situation and the FAIR principles [6].

\section{Results and Discussion}

Our analysis shows that the FAIR data principles are not explicitly mentioned, but the underlying equivalent concepts are indeed covered. The overall FAIR equivalence score is $43,79 \%$ (Table 1$)$. The scores per document show a great variation from $0(0 \%)$ to 14 $(93.3 \%)$. The analysis shows that the leadership is yet to make any provisions for the introduction and implementation of the FAIR data principles. However, the need for interoperability among heterogenous systems, has been comprehensively described.

Table 1. FAIR equivalence score for 14 documents with respect to the 15 facets of the FAIR data principles

\begin{tabular}{lll}
\hline FAIR data Principle (n=15) & Expected EQ max score & FAIR EQ score (\%) \\
\hline Findable (4) & 56 & $27(48.2)$ \\
Accessible (4) & 56 & $27(48.2)$ \\
Interoperable (3) & 42 & $20(47.6)$ \\
Reusable (4) & 56 & $18(28.52)$ \\
Total score & $\mathbf{2 1 0}$ & $\mathbf{9 2}(\mathbf{4 3 . 7 9 )}$ \\
\hline
\end{tabular}

\section{Conclusion}

Our evaluation reveals that there is no explicit uptake of the FAIR data principles in the health domain in Kenya. However, the equivalent of the FAIR concepts exists under a different name. We recommend that the leadership be offered a detailed introduction to the FAIR data principles and the steps necessary to FAIRify health data.

\section{References}

[1] Njeru I, Kareko D, Kisangau N, Langat D, Liku N, Owiso G, et al. Use of technology for public health surveillance reporting: opportunities, challenges and lessons learnt from Kenya. BMC Public Health. 2020;20(1):1101.

[2] Boeckhout M, Zielhuis GA, Bredenoord AL. The FAIR guiding principles for data stewardship: fair enough? European journal of human genetics. 2018;26(7):931-6.

[3] Jacobsen A, de Miranda Azevedo R, Juty N, Batista D, Coles S, Cornet R, et al. FAIR Principles: Interpretations and Implementation Considerations. Data Intelligence. 2020;2(1-2):10-29.

[4] Wilkinson MD, Dumontier M, Aalbersberg IJ, Appleton G, Axton M, Baak A, et al. The FAIR Guiding Principles for scientific data management and stewardship. Scientific Data. 2016;3(1):160018.

[5] Mons B. The VODAN IN: support of a FAIR-based infrastructure for COVID-19. European Journal of Human Genetics. 2020;28(6):724-7.

[6] van Reisen M, Oladipo F, Stokmans M, Mpezamihgo M, Folorunso S, Schultes E, et al. Design of a FAIR digital data health infrastructure in Africa for COVID-19 reporting and research. Advanced Genetics. 2021;2(2):e10050. 